\title{
Hierarchical functional nanoparticles boost osteoarthritis therapy by utilizing joint-resident mesenchymal stem cells
}

Yao Lu ${ }^{1,2^{*+}} \cdot$, Jieli Chen ${ }^{1+}$, Lihua $\mathrm{Li}^{3}$, Yumei Cao ${ }^{1}$, Yang Zhao ${ }^{1}$, Xiaoyu Nie ${ }^{1}$ and Changhai Ding ${ }^{1,4,5^{*}}$

\begin{abstract}
Utilization of joint-resident mesenchymal stem cells (MSC) to repair articular cartilage is a promising strategy in osteoarthritis (OA) therapy but remains a considerable research challenge. Here, hierarchical targeting and microenvironment responsive peptide functionalized nanoparticles (NPs) are used to achieve cartilage repair in situ. Ultrasmall copper oxide (CUO) NPs are conjugated with type 2 collagen and MSC dual-targeting peptide (designated WPV) with a matrix metalloproteinase 2 (MMP-2)-sensitive sequence as a spacer to achieve hierarchical targeting. Guided by this peptide, WPV-CuO NPs initially penetrate cartilage and subsequently expose the inner MSC-targeted peptide to attract MSCs through MMP-2 clearance. CuO further promotes chondrogenesis of MSCs. In an anterior cruciate ligament transection rat model, intraarticular injection of WPV-CuO NPs induces significant reduction of cartilage destruction. The therapeutic mechanism involves inhibition of the PI3K/AKT/mTOR pathway, as determined via transcriptome analysis. In conclusion, a novel therapeutic strategy for OA has been successfully developed based on localized MSC recruitment and cartilage repair without transplantation of exogenous cells or growth factors.
\end{abstract}

Keywords: Nanoparticles, Peptide, Osteoarthritis, Joint-resident mesenchymal stem cells, PI3KJAKT/mTOR

\footnotetext{
*Correspondence: oayul@smu.edu.cn; changhai.ding@utas.edu.au

${ }^{\dagger}$ Yao Lu and Jieli Chen have equally contributed to this work

${ }^{1}$ Clinical Research Center, Orthopedic Center, Zhujiang Hospital, Southern Medical University, Guangzhou, Guangdong 510282, China

Full list of author information is available at the end of the article
}

(c) The Author(s) 2022. Open Access This article is licensed under a Creative Commons Attribution 4.0 International License, which permits use, sharing, adaptation, distribution and reproduction in any medium or format, as long as you give appropriate credit to the original author(s) and the source, provide a link to the Creative Commons licence, and indicate if changes were made. The images or other third party material in this article are included in the article's Creative Commons licence, unless indicated otherwise in a credit line to the material. If material is not included in the article's Creative Commons licence and your intended use is not permitted by statutory regulation or exceeds the permitted use, you will need to obtain permission directly from the copyright holder. To view a copy of this licence, visit http://creativecommons.org/licenses/by/4.0/. The Creative Commons Public Domain Dedication waiver (http://creativeco mmons.org/publicdomain/zero/1.0/) applies to the data made available in this article, unless otherwise stated in a credit line to the data. 


\section{Graphical Abstract}
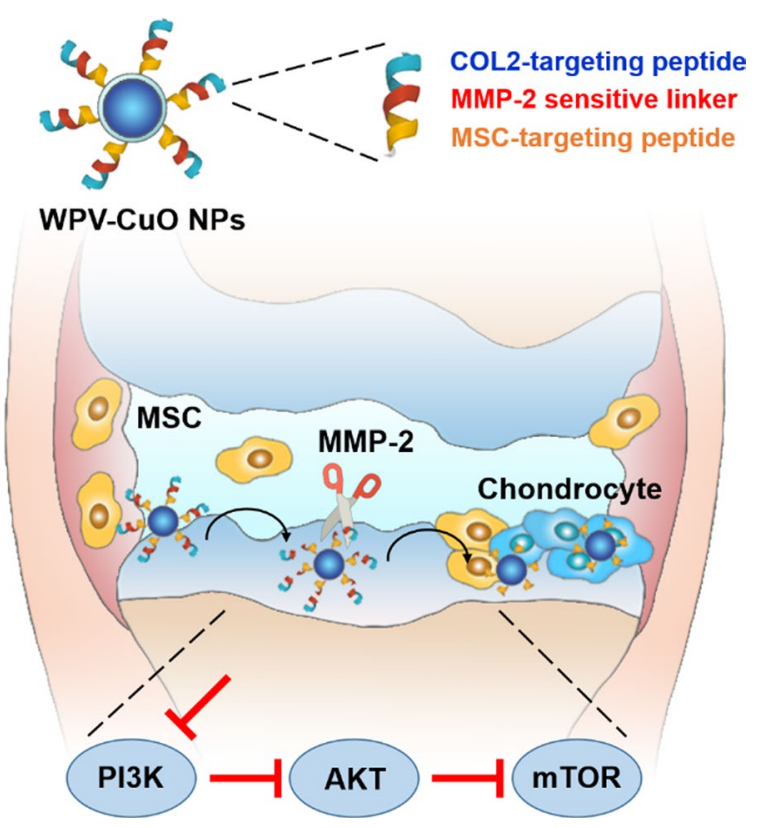

\section{Introduction}

Osteoarthritis (OA), the most common degenerative joint disease and the leading cause of adult disability, affects 250 million people worldwide [1]. With the quickening of the aging population process, the rising prevalence of OA will no doubt increase the burden with the disease, becoming a huge global public health challenge for the coming decades [2, 3]. Unfortunately, current pharmacological recommendations for OA therapy are restricted, thus the FDA distinguishes OA as a "serious disease with an unmet medical need" [4].

Analgesic (e.g. paracetamol) and non-steroidal antiinflammatory drugs (NSAIDs) are commonly used in OA patients for pain reduction and function improvement. However, these drugs provide limited long-term efficacy but raise the risk of gastrointestinal, cardiovascular, liver, and renal adverse events [5-7]. Intraarticular administrations, such as corticosteroids and hyaluronic acid, are also wildly used for the treatment of knee OA to reduce risks of systemic adverse events. However, the effects of intraarticular treatments on symptom relief and structural modification are still uncertain due to the considerable heterogeneity in clinical investigations [8-10]. Cartilage degradation is the most important hallmark of OA, and thus it is a major therapeutic target for the development of disease-modifying OA drugs (DMOADs) [4]. In recent years, sprifermin, a recombinant human fibroblast growth factor 18 (rhFGF18), emerges as a promising DMOAD [11]. Intraarticular injection of sprifermin shows a tendency of positive effects on reduction of cartilage loss, but the clinical significance is still undetermined due to the need of long follow-up periods to detect an effect on joint destruction [12-14]. Other potential DMOADs, such as vitamin D [15], anti-nerve growth factor neutralizing antibodies (e.g. tanezumab) [16], and transforming growth factor- $\beta$ [17], have been intensively investigated but not used in clinical OA treatment. Overall, current pharmacological interventions mainly alleviate the symptoms temporarily but fail to reverse the basic pathology of OA; namely clinical used drugs are not efficient enough to repair damaged articular cartilage.

Although it is generally believed that damaged cartilage cannot regenerate due to limited endogenous cells [18], mesenchymal stem cells (MSC) are relatively abundant within the joint regions, including synovium, synovial fluid, and adipose tissue [19], that potentially participate in cartilage repair during OA progression [19-21]. In clinic, microfracture surgery is applied to enhancing chondral resurfacing by taking advantage of bone marrow MSCs stimulation [22]. However, bone marrow-resident MSCs only start a chondrogenesis program when bone fracture occurs in adult [23]. Further, microfracture often results in the formation of fibrocartilage with weaker biochemistry and biomechanism rather than natural hyaline articular cartilage [24]. Hence an efficient and facile OA therapy based on joint-resident MSCs is highly desired but remain a challenge. To our knowledge, 
almost no efforts have been made to utilize joint-resident MSCs for cartilage damage repair in OA therapy to date despite microfracture surgery [25-27]. We aimed to recruit joint-resident MSCs and induce differentiation into chondrocytes to facilitate repair of articular cartilage using nanoparticles (NPs) without the requirement for surgery and exogenous cell transplantation.

Toward this goal, here we prepared ultrasmall copper oxide $(\mathrm{CuO})$ NPs and functionalized them with a hierarchical targeting peptide. We choose $\mathrm{Cu}$-based NPs because earlier studies by our group on $\mathrm{Cu}$-containing materials have demonstrated that $\mathrm{Cu}$ promotes chondrogenesis of MSCs and enhances cartilage formation $[28,29]$. The functional peptide (designated WPV) was designed to be type 2 collagen (COL2) and MSC dual-targeted (favoring cartilage penetration and MSCs recruitment, respectively), and a matrix metalloproteinase 2 (MMP-2)-sensitive sequence was inserted in the peptide as a spacer to achieve OA-related microenvironment responsive. The resultant WPV-CuO NPs were administered to anterior cruciate ligament transection (ACLT) rat model by intraarticular injection. This peptide-guided NPs could actively target cartilage, followed by its breakdown by abundant MMP-2 in the OA microenvironment, leading to exposure of the inner MSC-targeting peptide for MSC recruitment. Subsequently, the recruited MSCs were chondrogenically induced into chondrocytes by $\mathrm{CuO}$ NPs, achieving an efficient OA therapy via cartilage regeneration (Fig. 1).

\section{Materials and methods Preparation of CuO NPs}

We synthesized $\mathrm{CuO}$ NPs according to a previously reported method with modifications [30]. Briefly, $\mathrm{CuCl}_{2}$ solution $(10 \mathrm{mM}, 50 \mathrm{~mL})$ was heated to $80^{\circ} \mathrm{C}$ for $10 \mathrm{~min}$ and Lascorbic acid aqueous solution $(100 \mathrm{mM}, 50 \mathrm{~mL})$ added with constant stirring. The $\mathrm{pH}$ of the mixture was adjusted to 8.0-9.0 using $\mathrm{NaOH}$ solution $(1 \mathrm{M})$ and stirred at $80^{\circ} \mathrm{C}$ for $12 \mathrm{~h}$. The resultant solution was centrifuged and dialyzed to obtain CuO NPs.

\section{Preparation of hierarchical targeting CuO NPs}

For carboxyl group modification, $10 \mathrm{mg} \mathrm{CuO} \mathrm{NPs} \mathrm{were}$ reacted with $10 \mathrm{mg}$ polyacrylic acid (PAA) at $60^{\circ} \mathrm{C}$ for $2 \mathrm{~h}$ under stirring. The resultant $\mathrm{PAA}-\mathrm{CuO}(\mathrm{CuO}-\mathrm{COOH})$ NPs were dialyzed against $d_{d H_{2}} \mathrm{O}$ to remove excess PAA.

To prepare hierarchical functional NPs, a dual-targeted (COL2 and MSC) and MMP-2-cleavable peptide (WPV, see Additional file 1: Table S1) was designed and conjugated with $\mathrm{CuO}-\mathrm{COOH}$ NPs at a ratio of $1: 10$ via $N^{\prime}-$ ethylcarbodiimide hydrochloride/N-hydroxysuccinimide (EDC/NHS) reaction. A control peptide that could not be cleaved by MMP-2 (WGV, see Additional file 1: Table S1)

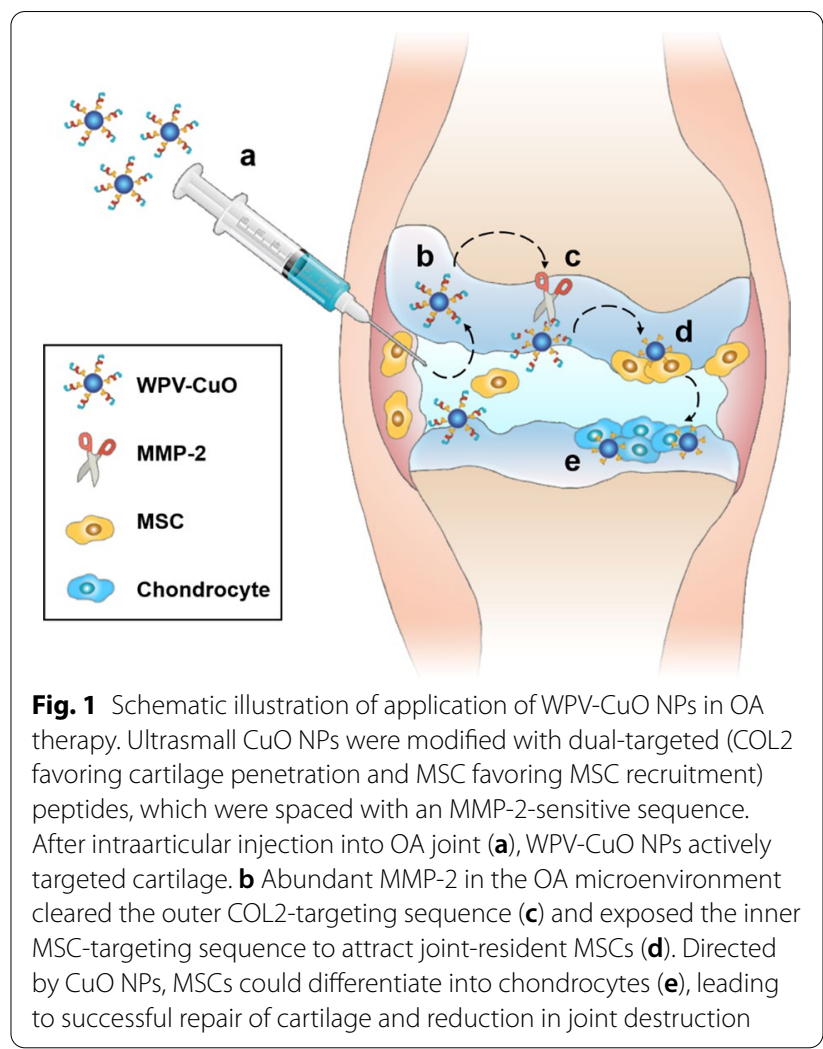

was used to prepare control NPs (termed WGV-CuO) using the same method. Another non-cartilage control peptide (LPV, see Additional file 1: Table S1) was used to prepare LPV-NPs. An $\mathrm{NH}_{2}$-rich linker (GGGGKKKK) was added to the $\mathrm{C}$-terminal end of the peptide to facilitate cross-linking with $\mathrm{CuO}-\mathrm{COOH}$ NPs. The conjugation rate of the peptide was measured by a BCA protein assay kit.

\section{Characterization of NPs}

The shapes and sizes of NPs were examined via highresolution transmission electron microscopy (HRTEM) and dynamic light scattering (DLS). X-ray diffraction (XRD) was employed to validate the crystalline structure. Changes in zeta potential, Fourier-transform infrared (FTIR) spectra and UV-vis absorption spectra were evaluated. High-performance liquid chromatography (HPLC) was performed to characterize MMP-2 sensitivity.

\section{Cytotoxicity}

Rat synovial MSCs were purchased from Shanghai Zhong Qiao Xin Zhou Biotechnology Co., Ltd. (China) and cultured in MSC medium (Shanghai Zhong Qiao Xin Zhou Biotechnology Co., Ltd.). To examine the cytotoxicity of NPs, MSCs were co-cultured with various concentrations 
of $\mathrm{CuO}$ NPs for $24 \mathrm{~h}$. The cell counting kit-8 (CCK-8) was used for evaluation of cell viability.

\section{MSC targeting}

SMSCs were co-cultured with $100 \mu \mathrm{g} \mathrm{mL}^{-1}$ Cy5.5-labeled WPV-CuO or WGV-CuO NPs and recombinant MMP-2 protein (30 $\mathrm{ng} \mathrm{mL}^{-1}$; PeproTech, USA). After co-culture for $24 \mathrm{~h}, \mathrm{MSCs}$ were washed with PBS to remove free $\mathrm{CuO}$ NPs and nuclei stained with 4',6-diamidino-2-phenylindole (DAPI; Sigma, USA). MSCs were examined under a fluorescence microscope (DMI4000, Leica) and analyzed with a flow cytometer (BD FACSCalibur, BD, USA).

\section{Chondrogenesis}

To assay chondrogenic gene expression, MSCs were prepared as above and cultured with chondrogenic medium (Cyagen Biosciences, USA) for 7 days. Relative mRNA expression levels of SOX6, aggrecan (ACAN), and COL2A1 were quantitatively determined via realtime polymerase chain reaction (RT-qPCR). Relative mRNA levels were normalized to that of $\beta$-actin using the $2^{-\triangle \triangle \mathrm{CT}}$ method. Primer sequences are described in Additional file 1: Table S2.

MSCs cultures were used for analysis of the chondrogenic inductivity of NPs. Briefly, $4 \times 10^{5}$ MSCs were centrifuged for $5 \mathrm{~min}$ at $500 \mathrm{~g}$ in a $15 \mathrm{~mL}$ tube and cultured for $24 \mathrm{~h}$ with chondrogenic medium to obtain a cell pellet, followed by the addition of NPs and MMP-2. After $24 \mathrm{~h}$ of culture, the medium was replaced with fresh chondrogenic medium. After 21 days, MSC pellets were fixed in $4 \%$ paraformaldehyde and embedded in epoxy resin for histological sectioning. Expression of COL2A1 was detected via immunohistochemistry.

\section{Cartilage targeting}

Male Sprague-Dawley (SD) rats (250-300g) were purchased from the Medical Experimental Animal Center of Guangdong Province. To establish the cartilage-targeting ability of our newly generated NPs, Cy5.5-labeled WPV-CuO NPs $\left(100 \mu \mathrm{L}, 1 \mathrm{mg} \mathrm{mL}^{-1}\right)$ were intraarticularly injected into the knee joint of rats. After 1, 4, and 24h, rats were euthanized and cartilage specimens from the knee joint were obtained. PBS and Cy5.5-labeled LPV$\mathrm{CuO}$ NPs $\left(100 \mu \mathrm{L}, 1 \mathrm{mg} \mathrm{mL}^{-1}\right)$ was administered to the control group. Samples were fixed and decalcified in ethylenediaminetetraacetic acid decalcifying solution (Leagene, China) for 6 weeks. Paraffin-embedded sections were prepared using a routine procedure followed by staining with DAPI. Finally, sections were imaged under a fluorescence microscope (BX51, Olympus, Japan).

\section{Therapeutic effects of NPs on ACLT rats}

An ACLT rat model was established [31] to assess the therapeutic efficacy of NPs against OA in vivo. To establish the abnormal mechanical loading-associated osteoarthritis model, anterior cruciate ligaments of the right knee joint of rats were transected. After 4 weeks, ACLT rats were randomly divided into three treatment groups ( $\mathrm{n}=6$ per group): (1) WPV-NPs, (2) WGV-NPs, and (3) PBS. NPs $\left(100 \mu \mathrm{L}, 1 \mathrm{mg} \mathrm{mL}^{-1}\right)$, and PBS $(100 \mu \mathrm{L})$ were intraarticularly injected into the right knee of ACLT rats following a q7dx4 course (4 times at intervals of 7 days). Sham-operated rats (performed by opening the joint capsule) were used as a control group. Rats were sacrificed at 8 weeks post operation and the right knee joints collected. Samples were fixed, decalcified, and stained with hematoxylin and eosin $(\mathrm{H} \& \mathrm{E})$ and safranin $\mathrm{O}$-Fast green. MMP-13 and COL2A1 levels were detected via immunofluorescence staining. Additionally, $H \& E$ staining of the main organs of rats was performed to evaluate the in vivo biosafety of NPs.

\section{Biodistribution of WPV-CuO NPs}

To investigate the accumulation of WPV-CuO NPs in knee and their metabolism in vivo, Cy5.5-labeled WPV$\mathrm{CuO}$ NPs were intraarticularly injected into the right knees of the OA rats. At day 1, 3, 5 and 10 post-injections, the main organs and knees of the OA rats were collected and imaged using a fluorescence imaging system (Bruker) at Ex: $630 \mathrm{~nm}, \mathrm{Em}: 700 \mathrm{~nm}$.

\section{Therapeutic mechanisms of action of WPV-CuO NPs against $O A$}

To explore the mechanisms underlying the therapeutic effects of WPV-CuO NPs, transcriptome analysis of ACLT rats was conducted. Established ACLT rats (4weeks post operation) were randomly divided into two groups ( $\mathrm{n}=3$ per group), specifically, WPV-CuO NPs and PBS, using the same treatment schedules specified above. After a 4-week treatment period, cartilage from the right knee joints was collected and total RNA extracted using TRIzol Reagent. The quality and quantity of RNA samples were determined with Agilent 2100 BioAnalyzer and Qubit RNA assay kits, respectively. mRNA libraries were generated by CapitalBio Technology Co., Ltd (Beijing, China) using Illumina HiSeq X10 (Illumina, USA) according to the manufacturer's instructions. The raw sequencing quality of each sample was determined with FastQC (v0.11.2) and Fastp (v0.14.0) programs. Tophat (v2.0.13) and Hisat2 (v2.1.0) software was utilized to map the trimmed sequence into the reference genome. Expression levels and differentially expressed genes (DEG) were determined via pairwise comparisons 
using the R statistical package software limma (v3.32.10). For functional and pathway enrichment analyses, DEGs with fold change $\geq 2, \mathrm{p}<0.05$ and false discovery rate $($ FDR $)<0.05$ were selected. KEGG functional enrichment analysis was performed using KOBAS (v3.0) to identify DEGs enriched in the pathway with significant features $(\mathrm{p}<0.05)$.

\section{Statistical analysis}

In vitro data are expressed as mean \pm standard deviation (SD) and in vivo data as mean \pm standard error of the mean (SEM). The two-sided Student's t-test was used for analyses.

\section{Results and discussion}

\section{Design, preparation, and characterization of the NPs}

In our design, NPs should first penetrate cartilage and subsequently recruit MSCs for repair. Given that NPs larger than $96 \mathrm{~nm}$ can barely penetrate the cartilage surface [32], we initially synthesized ultrasmall $\mathrm{CuO}$ NPs $\sim 3 \mathrm{~nm}$ in size (determined via TEM and DLS; Fig. 2b-d). NPs were modified with polyacrylic acid (PAA) to obtain $\mathrm{CuO}-\mathrm{COOH}$ NPs. To achieve hierarchical functionality, we combined a COL2-targeting peptide (WYRGRL) [32] with a MSC-targeting peptide (VTAMEPGQ) [33] to generate a dual peptide, which was further spaced with the matrix metalloproteinase 2 (MMP-2) clearance sequence

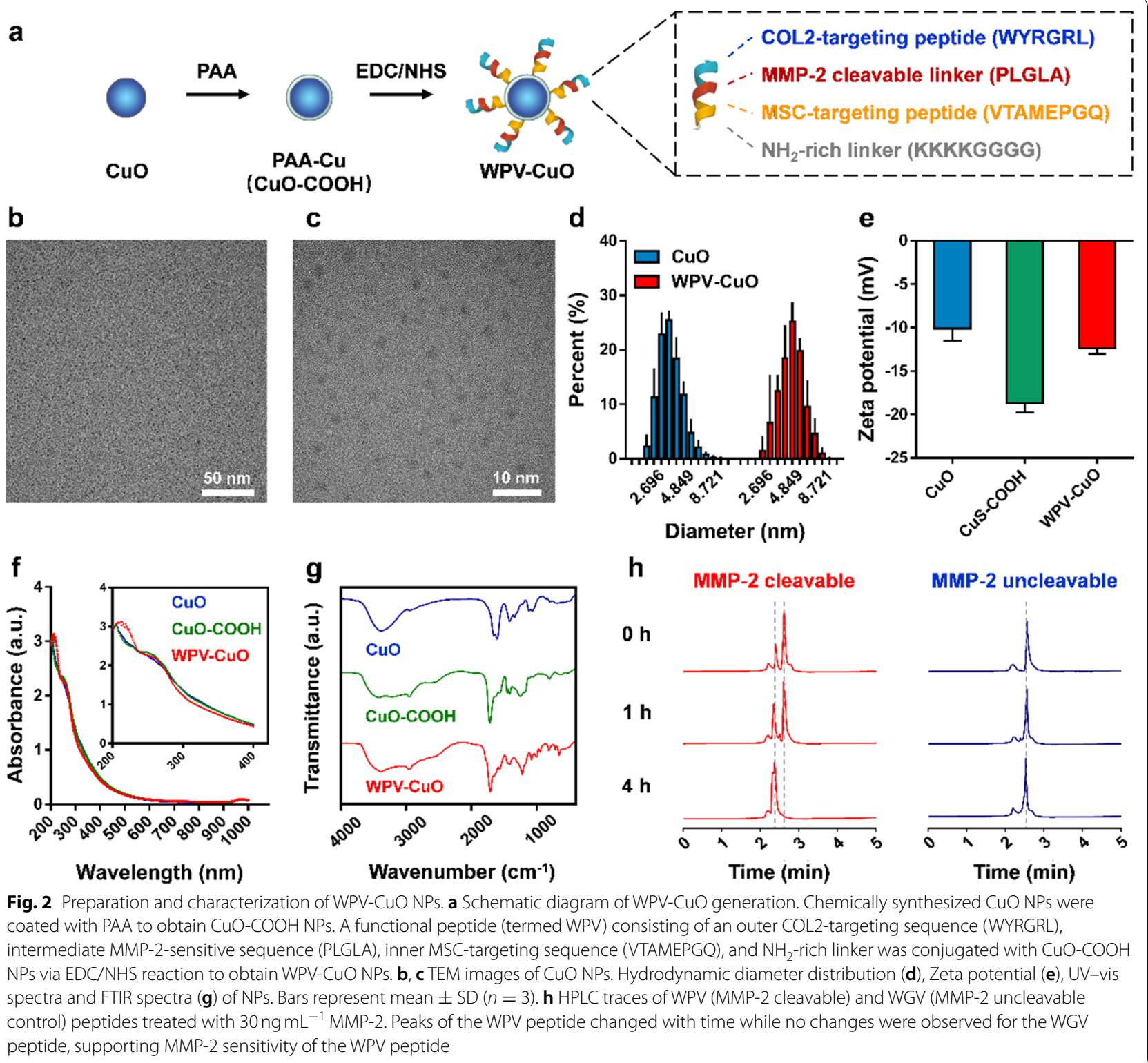


PLGLAG [34], designated WPV (Fig. 2a). Another dualtargeting peptide spaced with an adverse version of the MMP-2 sensitive sequence (GALGLP) was generated as an MMP-2 non-sensitive control, termed WGV (Additional file 1: Table S1). An $\mathrm{NH}_{2}$-rich linker, GGGGK$\mathrm{KKK}$, was added at the $\mathrm{C}$-terminal end of the peptide to facilitate cross-linking with $\mathrm{COOH}$ groups on NPs [35]. Finally, peptides were conjugated with $\mathrm{CuO}-\mathrm{COOH}$ NPs via an $N^{\prime}$-ethylcarbodiimide hydrochloride/ $N$-hydroxysuccinimide (EDC/NHS) reaction to obtain WPV-CuO and WGV-CuO NPs, respectively.

The size of the NPs slightly increased after peptide modification (Fig. 2d). Zeta potential of the NPs was decreased after $\mathrm{COOH}$ groups modification and then it was increased but remained negative after conjugated with the cationic peptides (Fig. 2e). Moreover, WPV-CuO exhibited a new absorption peak at $\sim 210 \mathrm{~nm}$ compared to $\mathrm{CuO}$ and $\mathrm{CuO}-\mathrm{COOH}$ NPs (Fig. 2f). Fourier transform infrared (FTIR) spectroscopy revealed that a new peak appeared in WPV-CuO NPs at $1568 \mathrm{~cm}^{-1}$, which corresponded to amide group in the peptide (Fig. 2g) [35]. These results confirmed successful conjugation of peptides to $\mathrm{CuO}$ NPs. The conjugation rate of the peptide on $\mathrm{CuO}$ NPs was approximately $94 \%$ (determined by a BCA method).

Peptides were further incubated with $30 \mathrm{ng} \mathrm{mL}^{-1}$ MMP-2 to stimulate the OA microenvironment and validate MMP-2-mediated breakdown [36]. High-performance liquid chromatography (HPLC) results confirmed cleavage of the MMP-2-sensitive WPV peptide in a time-dependent manner (Fig. 2h). In contrast, the peak intensity of MMP-2-insensitive WGV peptide was not changed in the presence of MMP-2, indicating that the negative control peptide is not cleavable by MMP- 2 .

\section{Hierarchical functions of the NPs}

To test the active cartilage-targeting effect of WPV-CuO NPs, Cy5.5-labeled WPV-CuO NPs $\left(100 \mu \mathrm{g} \mathrm{mL}^{-1}\right)$ and MMP-2 were intraarticularly injected into the knees of rats. NPs (red fluorescence) gradually accumulated in cartilage of the knee joints in a time-dependent manner (Fig. 3a). NPs clearly penetrated cartilage tissue after $4 \mathrm{~h}$ post-injection and remained distributed within the cartilage matrix after $24 \mathrm{~h}$. In contrast, red fluorescence was negligible in the cartilage after $24 \mathrm{~h}$ of intraarticular injection of the non-cartilage-targeting LPV-CuO NPs (Additional file 1: Fig. S1). These results demonstrate that WPV-CuO NPs actively target cartilage before clearance of the COL2-targeting peptide by MMP-2 in vivo.

Next, we examined whether the inner MSC-targeting sequence is effectively exposed after clearance of the MMP-2-sensitive linker. Synovial MSCs (SMSC, the main type of joint-resident MSCs involved in cartilage repair)
[19] were co-cultured with Cy5.5-labeled WPV-CuO and WGV-CuO NPs in the presence of MMP-2, respectively. Notably, strong red fluorescence was observed in SMSCs co-cultured with WPV-CuO NPs (Fig. 3b) while red fluorescence was weak in the WGV-CuO NP group. Flow cytometry analysis further confirmed significantly higher fluorescence intensity of WPV-CuO relative to the WGV-CuO and control groups (Fig. 3c; Additional file 1: Fig. S2).

\section{MSCs recruitment by the NPs}

Rat tibial plateau specimens were collected and co-cultured with WPV-CuO or WGV-CuO NPs in the presence of MMP-2. After 24h, SMSCs were seeded on tibial tissues and co-cultured for an additional $24 \mathrm{~h}$. Large numbers of SMSCs (red arrows) were attached on tibial plateaus treated with WPV-CuO NPs (observed via scanning electron microscopy (SEM); Fig. 3d) while limited SMSCs were detected in WGV-CuO NP and control groups (Fig. 3e). Our findings suggest that WPV-CuO NPs enhance SMSC recruitment through exposure of the inner MSC targeting sequence after breakdown of the MMP-2-sensitive linker. In contrast, WGV-CuO NPs could not attract SMSCs because the inner MSC targeting sequences were not exposed. Thus, WPV-CuO NPs newly developed in this study can successfully promote MSC recruitment via enhancement of MSC adhesion by the inner targeting sequence [37].

\section{Chondroinductivity of the NPs}

Guidance of MSC differentiation into cartilage after recruitment is essential in stem cell-based therapy for $\mathrm{OA}$. Accordingly, the chondroinductive effect of $\mathrm{CuO}$ NPs on SMSCs was examined. To this end, SMSCs were co-cultured with WPV-CuO and WGV-CuO NPs at a non-toxic concentration of $100 \mu \mathrm{g} \mathrm{mL}^{-1}$, as determined via the cell counting kit-8 (CCK-8) assay (Additional file 1: Fig. S3). MMP-2 was added and free NPs removed after $24 \mathrm{~h}$. Levels of all chondrogenic genes, including SRY-box transcription factor 6 (SOX6), aggrecan (ACAN), and alpha-1 type II collagen (COL2A1), were significantly higher in NP groups relative to the control group after 7 days of culture (Fig. 3f). Bare $\mathrm{CuO}$ NPs also up-regulated chondrogenic gene expression of SMSCs (Additional file 1: Fig. S4). In particular, expressions of the early chondrogenic markers, SOX6, and cartilage matrix marker, COL2A1, were highest in the WPV-CuO groups. In addition, chondrogenic gene expressions were slightly higher in the WPV-CuO than WGV-CuO group, consistent with the finding that WPV-CuO NPs could effectively target SMSCs. COL2 protein expression in SMSC cell pellets was further investigated via immunohistochemistry (Fig. 3g). 


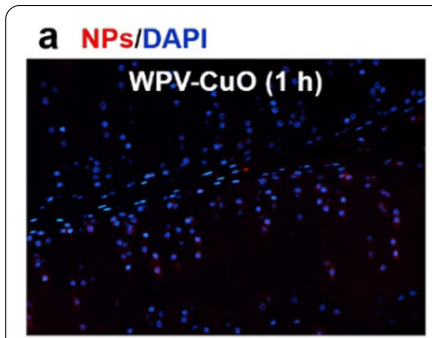

b NPS/DAPI
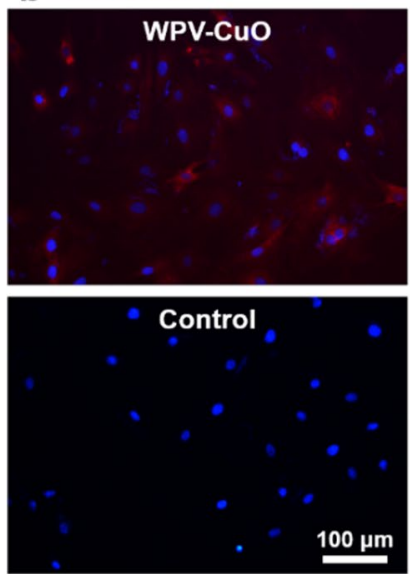

f

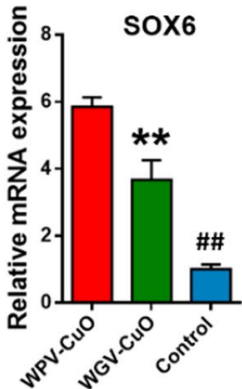

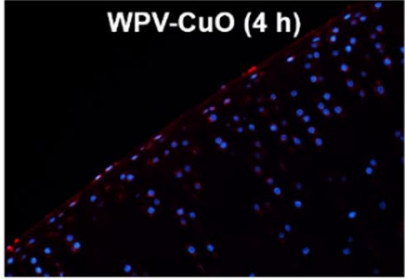

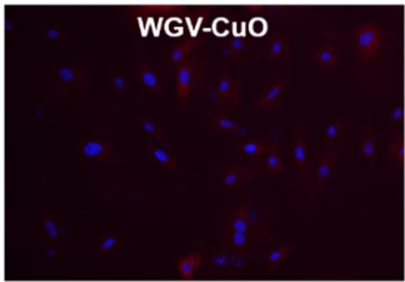

$C^{2000}$

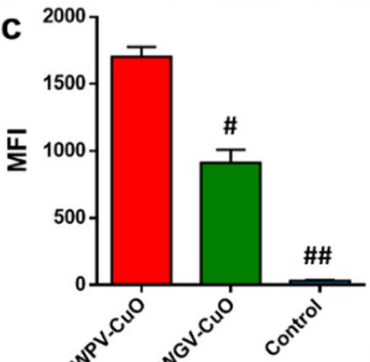

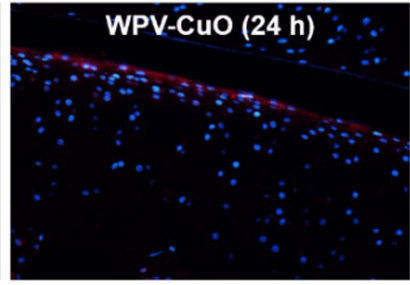

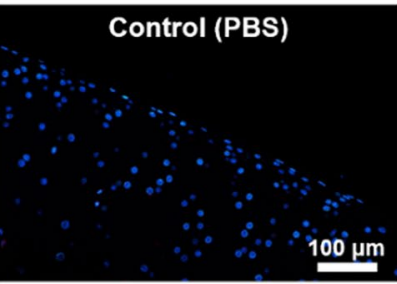

d
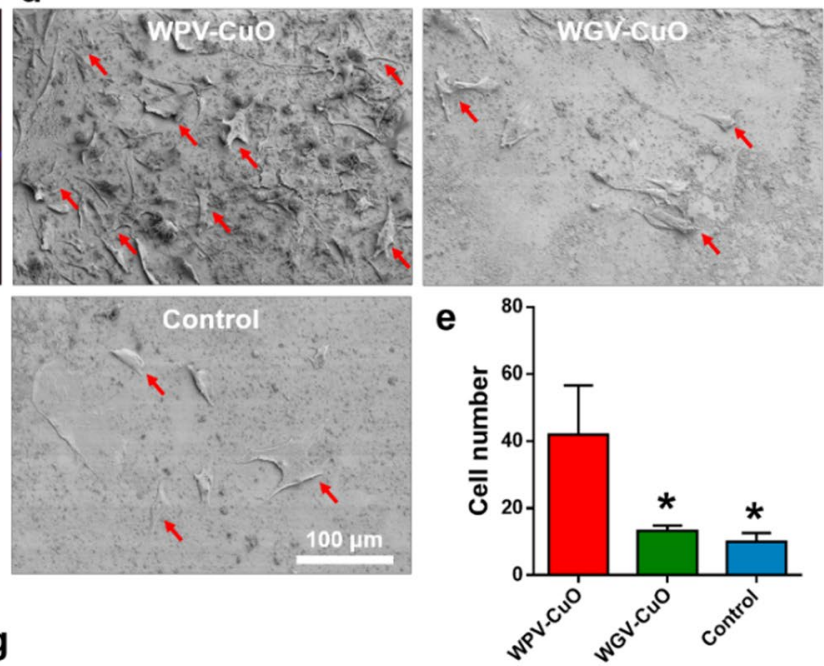

g
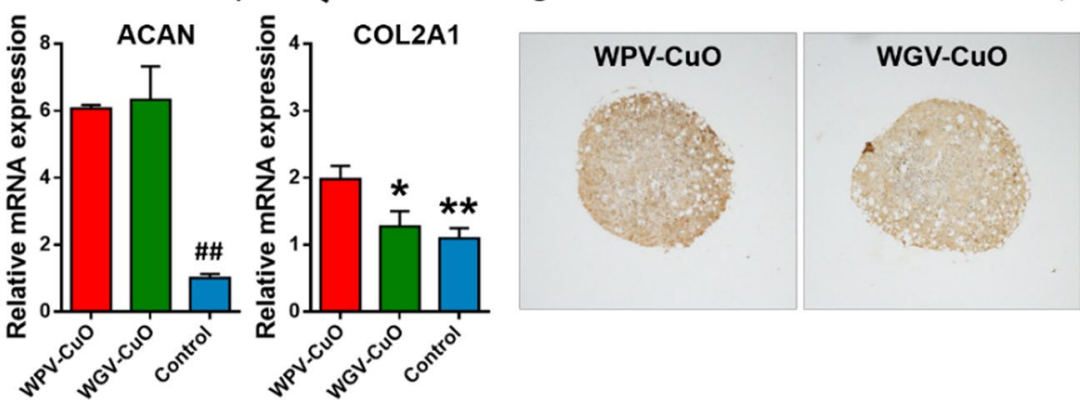

Control

$200 \mu \mathrm{m}$

Fig. 3 Dual targeting, SMSC recruitment, and chondroinductivity of NPs. a Fluorescence images of rat knee joint sections following intraarticular injection of Cy5.5-labled WPV-CuO NPs. WPV-CuO actively penetrated cartilage, even in the presence of MMP-2. Scale bar $=100 \mu \mathrm{m}$. b Fluorescence images of SMSCs treated with Cy5.5-labeled NPs and MMP-2. Scale bar $=100 \mu \mathrm{m}$. c Flow cytometry analysis showing highest mean fluorescence intensity (MFI) of SMSCs in the WPV-CuO NP group. Bars represent mean \pm SD $(n=3)$. d Representative SEM images of SMSC recruitment (highlighted by red arrows) in rat tibial plateau after treatment with NPs and MMP-2. e Calculation of the number of SMSCs attached to rat tibial plateau from five random SEM images. The data indicate that WPV-CUO NPs home into cartilage and expose the inner MSC targeting peptide due to MMP-2 sensitivity, leading to significant enhancement of SMSC adhesion to cartilage. Bars represent mean \pm SD $(n=5)$. $f$ Relative expression levels of chondrogenic genes in SMSCs co-cultured with NPs. Bars represent mean \pm SD $(n=3)$. $\mathbf{g}$ Immunohistological staining of COL2 in SMSC cell pellets co-cultured with NPs. Scale bar $=200 \mu \mathrm{m} .{ }^{*} p<0.05,{ }^{* *} p<0.01, \# p<0.05, \#$ \# $<0.01$

Cell pellets co-cultured with WPV-CuO NPs presented a deeper brown color compared to WGV-CuO and control groups, supporting chondroinductivity of WPV-CuO NPs. Our results support the functionality of hierarchical WPV-CuO NPs, which can effectively penetrate cartilage, recruit MSCs after clearance of the
MMP-2 sensitive linker, and induce differentiation of MSCs into chondrocytes.

\section{Therapeutic effect of the NPs against OA}

WPV-CuO NPs, WGV-CuO NPs and phosphate buffered solution (PBS) were intraarticularly injected following a q4dx7 course (four times at intervals of 7 days) 

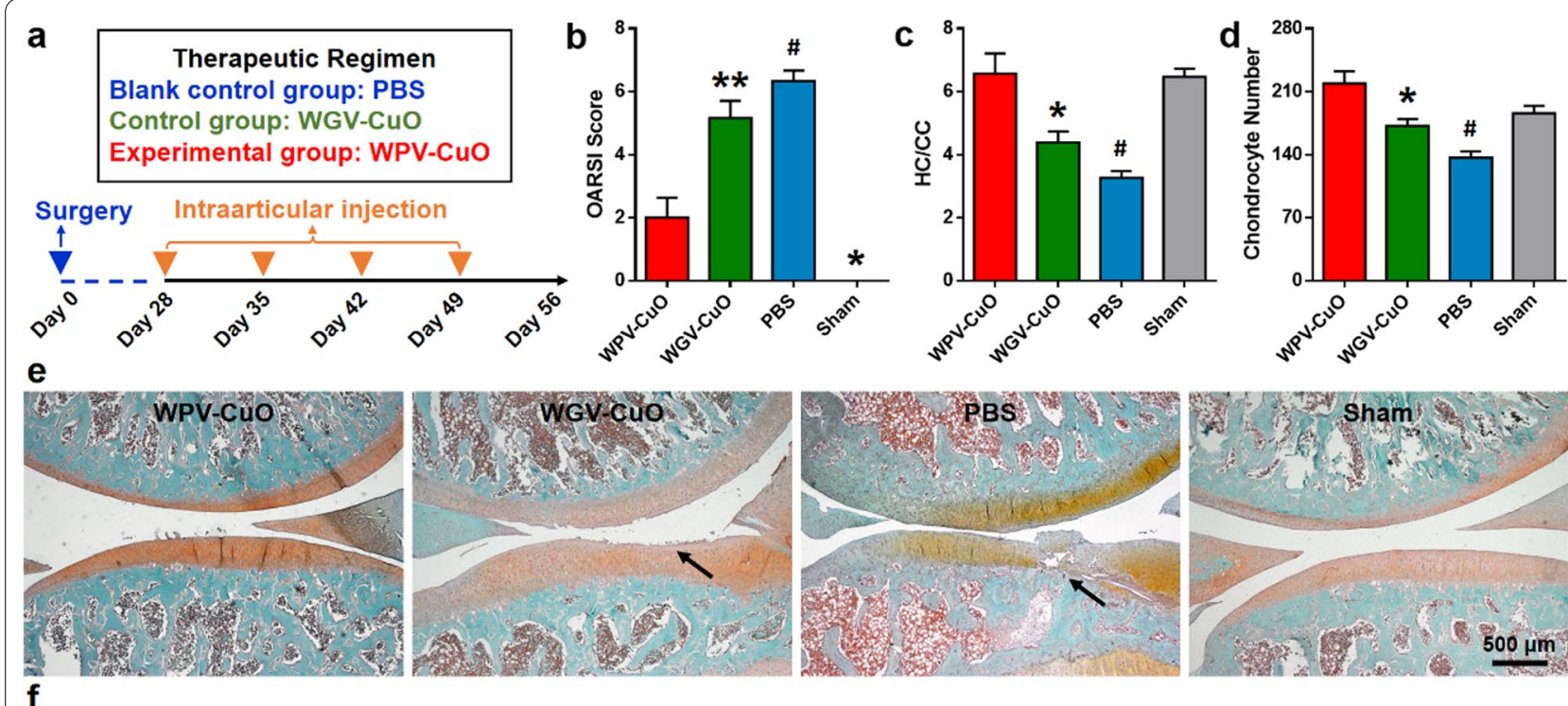

f
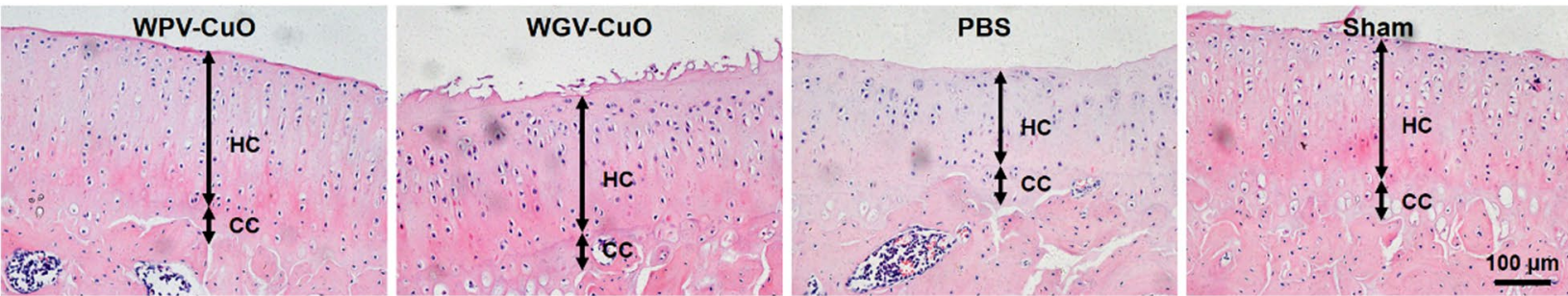

g COL2IDAPI
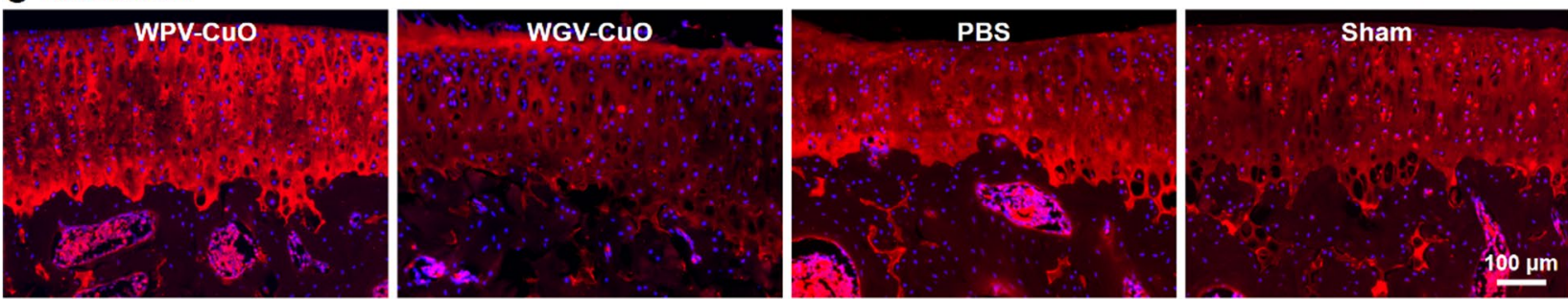

\section{h MMP-13/DAPI}
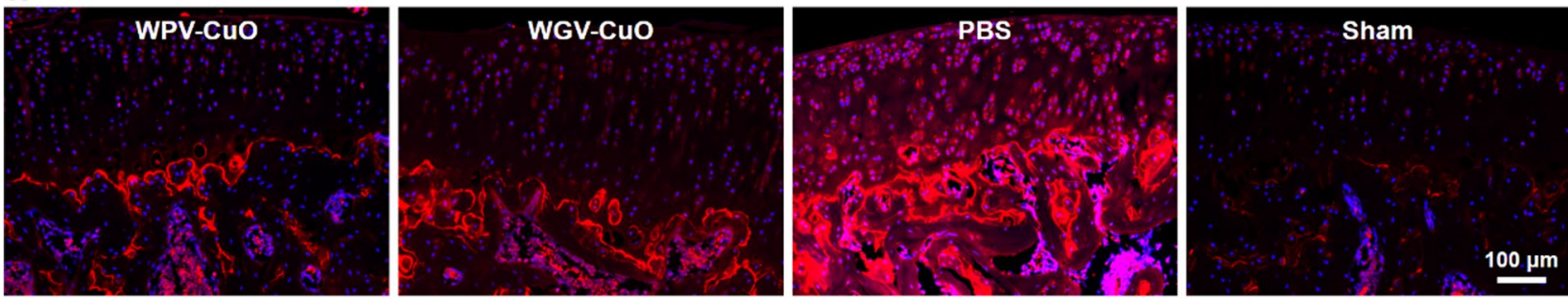

Fig. 4 Therapeutic effect of WPV-CUO NPs in an ACLT rat model. a Schematic diagram of surgical and different treatments. NPs or PBS were intraarticularly injected into the knee joint 4 weeks after establishment of the ACLT model following a $\mathrm{q} 7 \mathrm{~d} \times 4$ course. A sham treatment group was used as the control. $\mathbf{b}$ Cartilage erosion evaluation of knee joint sections based on OARSI score. $\mathbf{c}$ Ratio of hyaline cartilage and calcified cartilage $(\mathrm{HC} / \mathrm{CC})$. d The number of chondrocytes in the cartilage lesion area. Chondrocytes were calculated from the H\&E-stained sections shown in panel f. The WPV-CuO NP group presented the lowest OARSI score but highest HC/CC ratio among all treatment groups, indicating that WPV-CuO NPS reduce cartilage damage via generation of hyaline cartilage. Representative safranin O-fast green- $(\mathbf{e})$ and H\&E-stained (f) sections subjected to different treatments. Cartilage erosion is highlighted with black arrows in $\mathbf{d}$. Scale bar $=500 \mu \mathrm{m}$ in $\mathrm{d}$, and $=100 \mu \mathrm{m}$ in e. Immunofluorescent staining of COL2 $(\mathbf{g})$ and MMP-13 (h) in cartilage. Bars represent mean $\pm \operatorname{SEM}(n=6)$. ${ }^{*} p<0.05,{ }^{* *} p<0.01, \# p<0.05, \# \# p<0.01$ 
into knee joints of ACLT rats (Fig. 4a). Notably, WPV$\mathrm{CuO}$ NPs exerted a good therapeutic effect compared to WGV-CuO and PBS groups. Safranin O staining revealed no obvious cartilage damage in WPV-CuO NP-treated ACLT rats (Fig. 4e). In contrast, full-thickness cartilage damage was detected in PBS groups (black arrow). WGV-CuO NPs that lacked the ability to cleave MMP-2 could not expose the MSC targeting sequence but still displayed cartilage homing and chondrogenic properties and also reduced cartilage damage to some extent. Articular cartilage degeneration after different treatments was further graded using the Osteoarthritis Research Society International (OARSI)-modified score (Fig. 4b). The OARSI score of the WPV-CuO group was lowest among all treatment groups and was similar to that of the sham group, indicating that WPV-CuO NPs have the ability to repair cartilage erosion.

Hematoxylin and eosin (H\&E) staining disclosed similar thickness of hyaline cartilage (HC) between WPV$\mathrm{CuO} \mathrm{NP}$ and sham groups whereas $\mathrm{HC}$ was obviously degenerated and thinner in WGV-CuO NP and PBS groups (Fig. 4f). Overall, the hyaline cartilage: calcified cartilage $(\mathrm{HC} / \mathrm{CC})$ ratio was significantly decreased in WGV-CuO NP and PBS groups (Fig. 4c). The number of chondrocytes in the cartilage lesion area was significantly decreased in PBS group compared to sham group. Remarkably, the number of chondrocytes increased after WPV-CuO treatment (Fig. 4d). Immunofluorescent staining (Fig. 4g) indicated that COL2 expression was highest in WPV-CuO NP group. Micro-CT scan showed that WPV-CuO NPs slightly inhibited subchondral sclerosis, a typical feature of late-stage OA, compared to PBS group. However, the total bone value and trabecular bone structure had no significant difference between these two groups (Additional file 1: Fig. S5). The results suggest that WPV-CuO NPs exert therapeutic effects against OA and reduce cartilage destruction by promoting generation of hyaline cartilage. MMP13, a key cartilage-degrading enzyme in OA [38], was more highly expressed in WGV$\mathrm{CuO}$ NP and PBS groups compared to WPV-CuO NP and sham groups (Fig. 4h). Data from H\&E staining after treatment disclosed no adverse effects of NPs on the main organs of ACLT rats (Additional file 1: Fig. S6).

In addition, we intraarticularly injected Cy5.5-labeled WPV-CuO NPs into OA rats and investigated their metabolism using an in vivo fluorescence imaging system (Additional file 1: Fig. S7). The fluorescence intensity of the right knee gradually decreased over time and WPV-CuO NPs could accumulated in the right knee for approximately 10 days after injection. Moreover, the fluorescence intensity of liver and kidney was stronger than those of the other organs. The results suggest that WPV$\mathrm{CuO}$ NPs are mainly metabolized by the liver and kidney, which is consistent with previous reports that small nanomaterials can be excreted from the body by hepatic metabolism and renal clearance $[39,40]$.

\section{Therapeutic mechanisms of the NPs against OA}

To further clarify the mechanisms underlying activity of WPV-CuO NPs against OA, transcriptome analysis of collected cartilage from knee joints of ACLT rats after treatment was performed. Heatmap revealed the significant differentially expressed genes (DEG) between WPV$\mathrm{CuO}$ NP- and PBS-treated ACLT rats (Fig. 5a). Overall, 579 genes were upregulated and 571 genes were downregulated in the WPV-CuO NP group relative to the PBS group (Volcano plots, Fig. 4b).

Kyoto Encyclopedia of Genes and Genomes (KEGG) pathway enrichment analysis suggested that phosphoinositide 3-kinase/protein kinase B (PI3K/AKT) and mammalian target of rapamycin (mTOR) signaling pathways are highly related to the therapeutic mechanisms (Fig. 5c). Previous studies have demonstrated that activation of $\mathrm{PI} 3 \mathrm{~K} / \mathrm{AKT} / \mathrm{mTOR}$ enhances MMP production but reduces autophagy of chondrocytes, thereby accelerating cartilage destruction in OA [41-43]. Notably, PI3K and AKT as well as phosphorylated AKT (p-AKT) were significantly decreased in the WPV-CuO NP group, indicating suppression of the PI3K/AKT pathway (Fig. $5 \mathrm{~d}-\mathrm{f}$ ). Moreover, downstream mTOR expression was significantly inhibited in the WPV-CuO treatment group (Fig. 5g). Our results are consistent with recent literature reports that MSC transplantation suppresses PI3K/AKT/ mTOR to ameliorate tissue injury [44-46]. Therefore, the potential therapeutic mechanism underlying the activity of WPV-CuO NP against OA may be summarized as follows: WPV-CuO NPs recruit joint-resident MSCs and induce differentiation into chondrocytes, promoting repair of damaged cartilage and reduction of cartilage destruction through inhibition of the PI3K/AKT/mTOR pathway.

\section{Conclusions}

In summary, we have developed a facile peptide-modified NP strategy that takes advantage of joint-resident MSCs to achieve efficient OA therapy. The hierarchical functional peptide WPV facilitates active homing to cartilage by $\mathrm{CuO}$ NPs, subsequent recruitment of MSCs and chondrogenic induction to repair cartilage in an OA-related MMP-2 abundant microenvironment. WPV-CuO NPmediated MSC therapy not only reduced joint destruction by generating hyaline cartilage but also inhibited the $\mathrm{PI} 3 \mathrm{~K} / \mathrm{AKT} / \mathrm{mTOR}$ pathway, thereby enhancing the therapeutic effect of OA in vivo. Our newly developed NPs provide a general and useful tool for treating many other types of arthritis, since cartilage erosion and presence of 


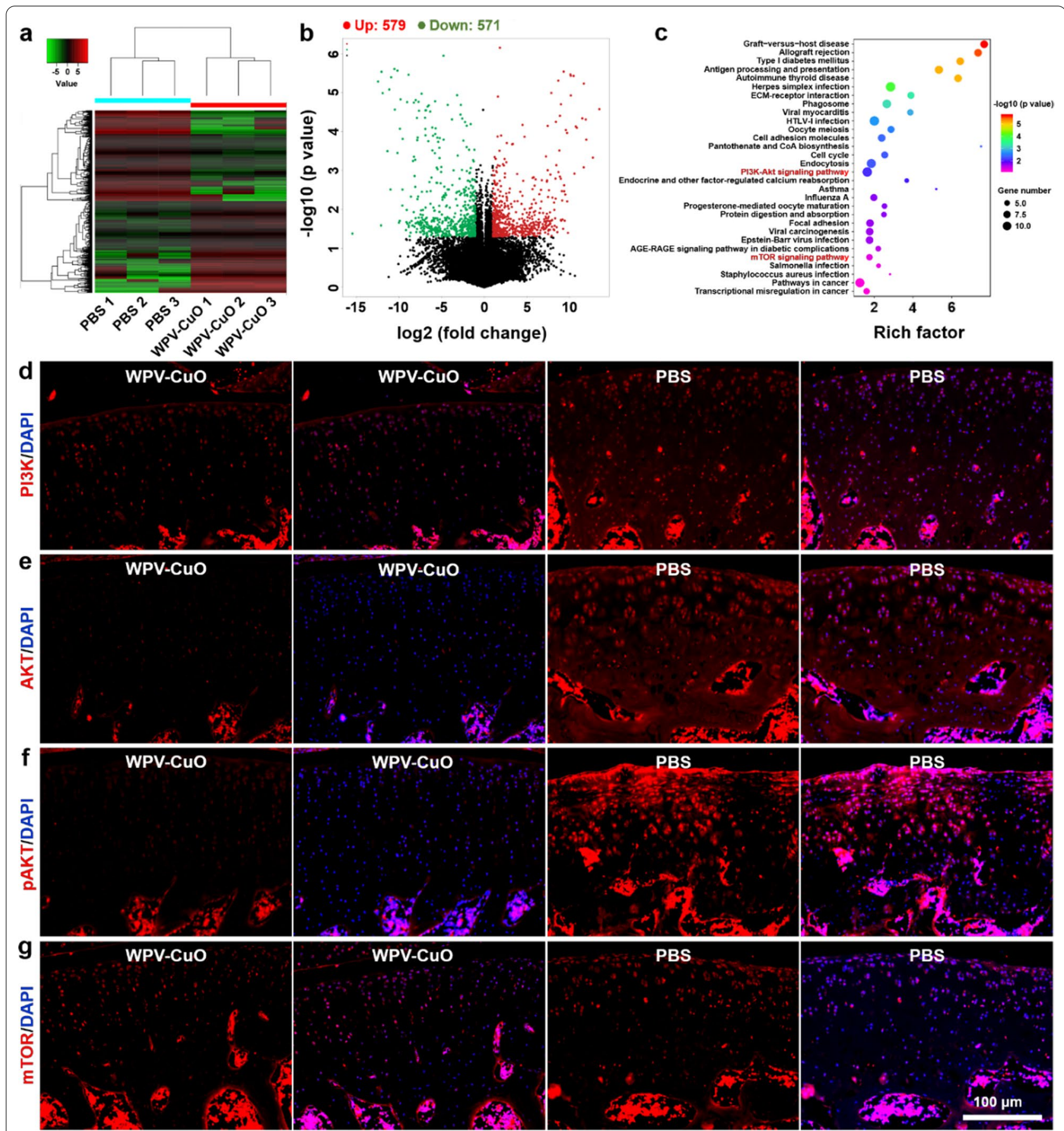

Fig. 5 Therapeutic mechanisms underlying activity of WPV-CuO NPs in OA. a Heatmap of significantly upregulated and downregulated genes between ACLT rats treated by PBS or WPV-CUO NPS. b Volcano plots showing significantly upregulated and downregulated genes following WPV-CuO treatment. Overall, 579 genes were up-regulated and 571 genes were down-regulated. c KEGG pathway enrichment analysis of the 30 most significantly enriched pathways. Immunofluorescent staining of PI3K (d), AKT (e), pAKT (f), and mTOR (g) expressed in cartilage after treatments. These data indicated that WPV-CuO NPs inhibited PI3K/AKT/mTOR pathway in OA model. Scale bar $=100 \mu \mathrm{m}$ 
MMP-2 are common features of degenerative and inflammatory joint diseases, such as rheumatoid arthritis [47], psoriatic arthritis [48], gouty arthritis [49], and systemic lupus erythematosus [50].

\section{Supplementary Information}

The online version contains supplementary material available at https://doi. org/10.1186/s12951-022-01297-w.

Additional file 1: Fig. S1. Cartilage-targeting effect of the control LPV$\mathrm{CuO}$ NPs (red fluorescence). Scale bar $=100 \mu \mathrm{m}$. Fig. S2. Flow cytometry analysis of SMSCS co-cultured with WPV-CUO, WGV-CUO, and PBS in the presence of MMP-2. Fig. S3. Cell viability of SMSCs co-cultured with CuO NPs after 24 h. Fig. S4. Chondrogenic inductivity of bare CuO NPs $(n=3)$. Fig. S5. Micro-CT scan of the joints of OA rats treated with WPV$\mathrm{CuO}$ or PBS $(n=6)$. Fig. S6. H\&E staining of the main organs of ACLT rats after different treatments.Scale bar $=500 \mu \mathrm{m}$. Fig. S7. Biodistribution of the Cy5.5-labeled WPV-CuO NPs after intraarticular injection. Table S1. Peptide properties. Table $\mathbf{S}$. Primers sequence used for RT-qPCR.

\section{Acknowledgements}

This study was supported by National Natural Science Foundation of China (81902198, 82172391), Guangdong Basic and Applied Basic Research Foundation (2020A1515010398), China National Postdoctoral Program for Innovative Talents (BX20190150), China Postdoctoral Science Foundation (2019M662980), and President Foundation of Zhujiang Hospital, Southern Medical University (yzjj2018rc09).

\section{Authors' contributions}

$Y L$ and $L L$ designed, prepared, and characterized the nanomaterials. $Y L$ and $C D$ designed the experiments. $Y L, J C, Y C, Y Z$, and $X N$ conducted all in vitro and in vivo experiments. $Y L$ wrote the manuscript. $C D$ revised the manuscript. All authors read and approved the final manuscript.

\section{Availability of data and materials}

All data generated or analyzed during this study are included in this published article and the Supporting Information.

\section{Declarations}

Ethics approval and consent to participate

All animal experimental protocols were approved by the Institutional Animal Care and Use Committee (IACUC) of General Hospital of Southern Theater Command of PLA (2020102001).

\section{Consent for publication}

All authors consent to publish.

\section{Competing interests}

The authors declare that they have no competing interests.

\section{Author details}

${ }^{1}$ Clinical Research Center, Orthopedic Center, Zhujiang Hospital, Southern Medical University, Guangzhou, Guangdong 510282, China. ${ }^{2}$ Guangdong Key Lab of Orthopedic Technology and Implant, General Hospital of Southern Theater Command of PLA, Guangzhou, Guangdong 510010, China. ${ }^{3}$ Department of Applied Physics, The Hong Kong Polytechnic University, Hung Hom, Kowloon, Hong Kong 999077, China. ${ }^{4}$ Guangdong Provincial Key Laboratory of Bone and Joint Degeneration Diseases, Academy of Orthopedics, Southern Medical University, Guangzhou 510630, China. ${ }^{5}$ Menzies Institute for Medical Research, University of Tasmania, Hobart, TAS 7000, Australia.

Received: 25 November 2021 Accepted: 2 February 2022 Published online: 19 February 2022

\section{References}

1. Hunter DJ, Bierma-Zeinstra S. Osteoarthritis. Lancet. 2019:393(10182):1745-59.

2. Coryell PR, Diekman BO, Loeser RF. Mechanisms and therapeutic implications of cellular senescence in osteoarthritis. Nat Rev Rheumatol. 2021;17(1):47-57.

3. Arden NK, Perry TA, Bannuru RR, Bruyère $O$, Cooper $C$, Haugen IK Hochberg MC, McAlindon TE, Mobasheri A, Reginster J-Y. Non-surgical management of knee osteoarthritis: comparison of ESCEO and OARSI 2019 guidelines. Nat Rev Rheumatol. 2021;17(1):59-66.

4. Latourte A, Kloppenburg M, Richette P. Emerging pharmaceutical therapies for osteoarthritis. Nat Rev Rheumatol. 2020;16(12):673-88.

5. Roberts E, Delgado Nunes V, Buckner S, Latchem S, Constanti M, Miller P, Doherty M, Zhang W, Birrell F, Porcheret M, Dziedzic K, Bernstein I, Wise E, Conaghan PG. Paracetamol: not as safe as we thought? A systematic literature review of observational studies. Ann Rheum Dis. 2016;75(3):552-9.

6. Nissen SE, Yeomans ND, Solomon DH, Lüscher TF, Libby P, Husni ME, Graham DY, Borer JS, Wisniewski LM, Wolski KE, Wang Q, Menon V, Ruschitzka F, Gaffney M, Beckerman B, Berger MF, Bao W, Lincoff AM. Cardiovascular safety of celecoxib, naproxen, or ibuprofen for arthritis. N Engl J Med. 2016;375(26):2519-29.

7. Bally M, Dendukuri N, Rich B, Nadeau L, Helin-Salmivaara A, Garbe E, Brophy JM. Risk of acute myocardial infarction with NSAIDs in real world use: bayesian meta-analysis of individual patient data. BMJ. 2017;357:j1909.

8. Sharma L. Osteoarthritis of the knee. N Engl J Med. 2021:384(1):51-9.

9. Angeles-Han ST, Ringold S, Beukelman T, Lovell D, Cuello CA, Becker ML, Colbert RA, Feldman BM, Holland GN, Ferguson PJ, Gewanter H, Guzman J, Horonjeff J, Nigrovic PA, Ombrello MJ, Passo MH, Stoll ML, Rabinovich CE, Sen HN, Schneider R, Halyabar O, Hays K, Shah AA, Sullivan N, Szymanski AM, Turgunbaev M, Turner A, Reston J. 2019 American college of rheumatology/arthritis foundation guideline for the screening, monitoring, and treatment of juvenile idiopathic arthritis-associated uveitis. Arthritis Rheumatol. 2019;71(6):864-77.

10. Hunter DJ. Viscosupplementation for osteoarthritis of the knee. N Engl J Med. 2015:372(11):1040-7.

11. Li J, Wang X, Ruan G, Zhu Z, Ding C. Sprifermin: a recombinant human fibroblast growth factor 18 for the treatment of knee osteoarthritis. Expert Opin Inv Drug. 2021;30(9):923-30.

12. Hochberg MC, Guermazi A, Guehring H, Aydemir A, Wax S, FleuranceauMorel P, Reinstrup Bihlet A, Byrjalsen I, Ragnar Andersen J, Eckstein F. Effect of intra-articular sprifermin vs placebo on femorotibial joint cartilage thickness in patients with osteoarthritis: the FORWARD randomized clinical trial. JAMA. 2019;322(14):1360-70.

13. Brett $A$, Bowes MA, Conaghan PG, Ladel C, Guehring H, Moreau F, Eckstein F. Automated MRI assessment confirms cartilage thickness modification in patients with knee osteoarthritis: post-hoc analysis from a phase II sprifermin study. Osteoarthr Cartil. 2020;28(11):1432-6.

14. Dahlberg LE, Aydemir A, Muurahainen N, Gühring $H$, Fredberg Edebo $H$ Krarup-Jensen N, Ladel CH, Jurvelin JS. A first-in-human, double-blind, randomised, placebo-controlled, dose ascending study of intra-articular rhFGF18 (sprifermin) in patients with advanced knee osteoarthritis. Clin Exp Rheumatol. 2016;34(3):445-50.

15. Jin X, Jones G, Cicuttini F, Wluka A, Zhu Z, Han W, Antony B, Wang X Winzenberg T, Blizzard L, Ding C. Effect of vitamin D supplementation on tibial cartilage volume and knee pain among patients with symptomatic knee osteoarthritis: a randomized clinical trial. JAMA. 2016;315(10):1005-13.

16. Lane NE, Schnitzer TJ, Birbara CA, Mokhtarani M, Shelton DL, Smith MD, Brown MT. Tanezumab for the treatment of pain from osteoarthritis of the knee. N Engl J Med. 2010;363(16):1521-31.

17. Lee B, Parvizi J, Bramlet D, Romness DW, Guermazi A, Noh M, Sodhi N, Khlopas A, Mont MA. Results of a phase II study to determine the efficacy and safety of genetically engineered allogeneic human chondrocytes expressing TGF-ß31. J Knee Surg. 2020;33(02):167-72.

18. Lu R, Zhang Y, Tao H, Zhou L, Li H, Chen T, Zhang P, Lu Y, Chen S. Gadolinium-hyaluronic acid nanoparticles as an efficient and safe magnetic resonance imaging contrast agent for articular cartilage injury detection. Bioact Mater. 2020;5(4)::758-767

19. McGonagle D, Baboolal TG, Jones E. Native joint-resident mesenchymal stem cells for cartilage repair in osteoarthritis. Nat Rev Rheumatol. 2017;13(12):719-30. 
20. Hunziker EB, Rosenberg LC. Repair of partial-thickness defects in articular cartilage: cell recruitment from the synovial membrane. J Bone Joint Surg Am. 1996;78(5):721-33.

21. Baboolal TG, Mastbergen SC, Jones E, Calder SJ, Lafeber FPJG, McGonagle D. Synovial fluid hyaluronan mediates MSC attachment to cartilage, a potential novel mechanism contributing to cartilage repair in osteoarthritis using knee joint distraction. Ann Rheum Dis. 2016;75(5):908-15.

22. Chen M, Li Y, Liu S, Feng Z, Wang H, Yang D, Guo W, Yuan Z, Gao S, Zhang Y, Zha K, Huang B, Wei F, Sang X, Tian Q, Yang X, sui X, Zhou Y, Zheng Y. Guo Q. Hierarchical macro-microporous WPU-ECM scaffolds combined with microfracture promote in situ articular cartilage regeneration in rabbits. Bioact Mater. 2021;6(7):1932-44

23. Einhorn TA, Gerstenfeld LC. Fracture healing: mechanisms and interventions. Nat Rev Rheumatol. 2015;11(1):45-54.

24. Makris EA, Gomoll AH, Malizos KN, Hu JC. Athanasiou, repair and tissue engineering techniques for articular cartilage. Nat Rev Rheumatol. 2015;11(1):21-34

25. Peat $\mathrm{G}$, Thomas MJ. Osteoarthritis year in review 2020: epidemiology \& therapy. Osteoarthr Cartil. 2021;29(2):180-9.

26. van den Bosch MHJ. Osteoarthritis year in review 2020: biology. Osteoarthr Cartil. 2021;29(2):143-50.

27. Gaharwar AK, Singh I, Khademhosseini A. Engineered biomaterials for in situ tissue regeneration. Nat Rev Mater. 2020;5(9):686-705.

28. Xu C, Chen J, Li L, Pu X, Chu X, Wang X, Li M, Lu Y, Zheng X. Promotion of chondrogenic differentiation of mesenchymal stem cells by copper: implications for new cartilage repair biomaterials. Mater Sci Eng C. 2018:93:106-14.

29. Lu Y, Li L, Lin Z, Wang L, Lin L, Li M, Zhang Y, Yin Q, Li Q, Xia H. A New treatment modality for rheumatoid arthritis: combined photothermal and photodynamic therapy using Cu7.2S4 nanoparticles. Adv Healthc Mater. 2018:7(14):1800013.

30. Liu T, Xiao B, Xiang F, Tan J, Chen Z, Zhang X, Wu C, Mao Z, Luo G, Chen $X$, Deng J. Ultrasmall copper-based nanoparticles for reactive oxygen species scavenging and alleviation of inflammation related diseases. Nat Commun. 2020;11(1):2788.

31. Cui Z, Crane J, Xie H, Jin X, Zhen G, Li C, Xie L, Wang L, Bian Q, Qiu T, Wan $M$, Xie M, Ding S, Yu B, Cao X. Halofuginone attenuates osteoarthritis by inhibition of TGF- $\beta$ activity and $\mathrm{H}$-type vessel formation in subchondral bone. Ann Rheum Dis. 2016;75(9):1714-21.

32. Rothenfluh DA, Bermudez $\mathrm{H}, \mathrm{O}^{\prime}$ Neil $\mathrm{CP}$, Hubbell JA. Biofunctional polymer nanoparticles for intra-articular targeting and retention in cartilage. Nat Mat. 2008;7(3):248-54.

33. Ma K, Wang D-D, Lin Y, Wang J, Petrenko V, Mao C. Synergetic targeted delivery of sleeping-beauty transposon system to mesenchymal stem cells using LPD nanoparticles modified with a phage-displayed targeting peptide. Adv Funct Mater. 2013;23(9):1172-81.

34. Trang VH, Zhang X, Yumul RC, Zeng W, Stone IJ, Wo SW, Dominguez MM, Cochran JH, Simmons JK, Ryan MC, Lyon RP, Senter PD, Levengood MR. A coiled-coil masking domain for selective activation of therapeutic antibodies. Nat Biotechnol. 2019;37(7):761-5.

35. Li L, Lu Y, Lin Z, Mao AS, Jiao J, Zhu Y, Jiang C, Yang Z, Peng M, Mao C. Ultralong tumor retention of theranostic nanoparticles with short peptide-enabled active tumor homing. Mater Horiz. 2019;6(9):1845-53.

36. Kim KS, Choi HM, Lee YA, Choi IA, Lee SH, Hong SJ, Yang HI, Yoo MC. Expression levels and association of gelatinases MMP-2 and MMP-9 and collagenases MMP-1 and MMP-13 with VEGF in synovial fluid of patients with arthritis. Rheumatol Int. 2011;31(4):543-7.

37. Ramaraju H, Miller SJ, Kohn DH. Dual-functioning peptides discovered by phage display increase the magnitude and specificity of BMSC attachment to mineralized biomaterials. Biomaterials. 2017:134:1-12.

38. Wang M, Sampson ER, Jin H, Li J, Ke QH, Im H-J, Chen D. MMP13 is a critical target gene during the progression of osteoarthritis. Arthritis Res Ther. 2013;15(1):R5

39. Wang B, He X, Zhang Z, Zhao Y, Feng W. Metabolism of nanomaterials in vivo: blood circulation and organ clearance. Acc Chem Res. 2013:46(3):761-9.

40. Lu Y, Li L, Du J, Chen J, Xu X, Yang X, Ding C, Mao C. Immunotherapy for tumor metastasis by artificial antigen-presenting cells via targeted microenvironment regulation and T-cell activation. ACS Appl Mater Interfaces. 2021;13(47):55890-901.
41. Zhang Y, Vasheghani F, Li Y-h, Blati M, Simeone K, Fahmi H, Lussier B, Roughley P, Lagares D, Pelletier J-P, Martel-Pelletier J, Kapoor M. Cartilagespecific deletion of mTOR upregulates autophagy and protects mice from osteoarthritis. Ann Rheum Dis. 2015;74(7):1432-40.

42. Huang X, Ni B, Mao Z, Xi Y, Chu X, Zhang R, Ma X, You H. NOV/CCN3 induces cartilage protection by inhibiting PI3K/AKT/mTOR pathway. J Cell Mol Med. 2019:23(11):7525-34.

43. Zhou X, Li J, Zhou Y, Yang Z, Yang H, Li D, Zhang J, Zhang Y, Xu N, Huang Y, Jiang L. Down-regulated ciRS-7/up-regulated miR-7 axis aggravated cartilage degradation and autophagy defection by PI3K/AKT/mTOR activation mediated by IL-17A in osteoarthritis. Aging. 2020;12(20):20163-83.

44. Xu X, Yu H, Sun L, Zheng C, Shan Y, Zhou Z, Wang C, Chen B. Adiposederived mesenchymal stem cells ameliorate dibutyltin dichlorideinduced chronic pancreatitis by inhibiting the PI3K/AKT/mTOR signaling pathway. Mol Med Rep. 2020;21 (4):1833-40.

45. Xiang J, Jiang T, Zhang W, Xie W, Tang X, Zhang J. Human umbilical cordderived mesenchymal stem cells enhanced HK-2 cell autophagy through MicroRNA-145 by inhibiting the PI3K/AKT/mTOR signaling pathway. Exp Cell Res. 2019;378(2):198-205.

46. He H, Zeng Q, Huang G, Lin Y, Lin H, Liu W, Lu P. Bone marrow mesenchymal stem cell transplantation exerts neuroprotective effects following cerebral ischemia/reperfusion injury by inhibiting autophagy via the PI3K/Akt pathway. Brain Res. 2019;1707:124-32.

47. Xue M, McKelvey K, Shen K, Minhas N, March L, Park S-Y, Jackson CJ. Endogenous MMP-9 and not MMP-2 promotes rheumatoid synovial fibroblast survival, inflammation and cartilage degradation. Rheumatology. 2014;53(12):2270-9.

48. Hitchon CA, Danning CL, Illei GG, El-Gabalawy HS, Boumpas DT. Gelatinase expression and activity in the synovium and skin of patients with erosive psoriatic arthritis. J Rheumatol. 2002;29(1):107-17.

49. Chu S-C, Yang S-F, Tzang B-S, Hsieh Y-S, Lue K-H, Lu K-H. Cathepsin B and cystatin $C$ play an inflammatory role in gouty arthritis of the knee. Clin Chim Acta. 2010;411(21):1788-92.

50. Makowski GS, Ramsby ML. Zymographic analysis of latent and activated forms of matrix metalloproteinase- 2 and -9 in synovial fluid: correlation to polymorphonuclear leukocyte infiltration and in response to infection. Clin Chim Acta. 2003;329(1):77-81.

\section{Publisher's Note}

Springer Nature remains neutral with regard to jurisdictional claims in published maps and institutional affiliations.

Ready to submit your research? Choose BMC and benefit from:

- fast, convenient online submission

- thorough peer review by experienced researchers in your field

- rapid publication on acceptance

- support for research data, including large and complex data types

- gold Open Access which fosters wider collaboration and increased citations

- maximum visibility for your research: over $100 \mathrm{M}$ website views per year

At BMC, research is always in progress.

Learn more biomedcentral.com/submissions 